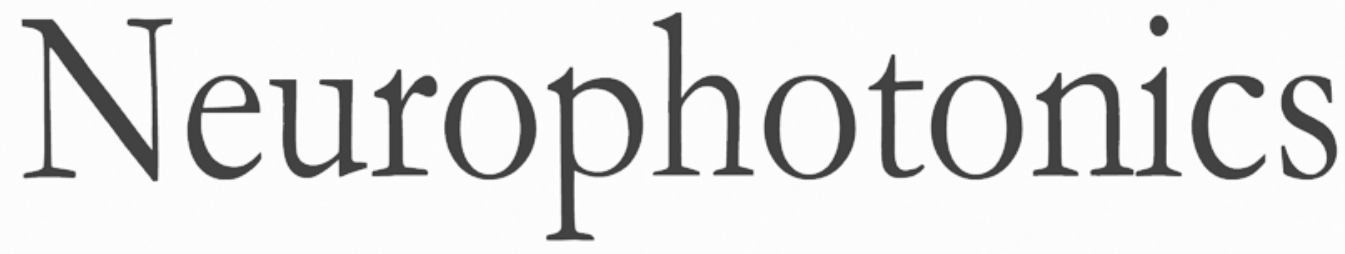

\title{
Cerebral oximetry in preterm infants: an agenda for research with a clear clinical goal
}

Gorm Greisen

Bjørn Andresen

Anne Mette Plomgaard

Simon Hyttel-Sørensen 


\title{
Cerebral oximetry in preterm infants: an agenda for research with a clear clinical goal
}

\author{
Gorm Greisen, ${ }^{*}$ Bjørn Andresen, Anne Mette Plomgaard, and Simon Hyttel-Sørensen \\ Rigshospitalet and Copenhagen University, Department of Neonatology, Blegdamsvej 9, 2100 Copenhagen Ø, Denmark
}

\begin{abstract}
Preterm birth constitutes a major cause of death before 5 years of age and it is a major cause of neurodevelopmental impairment across the world. Preterm infants are most unstable during the transition between fetal and newborn life during the first days of life and most brain damage occurs in this period. The brain of the preterm infant is accessible for tissue oximetry by near-infrared spectroscopy. Cerebral oximetry has the potential to improve the long-term outcome by helping to tailor the support of respiration and circulation to the individual infant's needs, but the evidence is still lacking. The goals for research include testing the benefit and harms of cerebral oximetry in large-scale randomized trials, improved definition of the hypoxic threshold, better understanding the effects of intensive care on cerebral oxygenation, as well as improved precision of oximeters and calibration among devices or standardization of values in the hypoxic range. These goals can be pursued in parallel. () The Authors. Published by SPIE under a Creative Commons Attribution 3.0 Unported License. Distribution or reproduction of this work in whole or in part requires full attribution of the original publication, including its DOI. [DOI: 10.1117/1.NPh.3.3.031407]
\end{abstract}

Keywords: near-infrared spectroscopy; brain hypoxia; preterm infant; brain injuries; clinical study.

Paper 16017SSR received Mar. 18, 2016; accepted for publication Mar. 22, 2016; published online Apr. 25, 2016.

\section{Introduction}

\subsection{First Studies in Preterm Infants}

Near-infrared spectroscopy (NIRS) was first applied to preterm infants 30 years ago by Brazy et al. ${ }^{1}$ and demonstrated meaningful correlations between apnea and arterial $\mathrm{pO}_{2}$ on one side and cerebral oxyhemoglobin and oxidized cytochrome $\mathrm{aa}_{3}$ concentrations measured by NIRS on the other side. The following year, a correlation between $\mathrm{PaCO}_{2}$ and cerebral blood volume and cerebral oxygenation index measured by NIRS was demonstrated $^{2}$ and 5 years later we could publish statistically strong correlations between cerebral blood flow measured by xenon clearance and cerebral blood volume and cytochrome $\mathrm{aa}_{3}$ oxidation as estimated by NIRS. ${ }^{3}$

Thus from the first applications, the preterm brain appeared perfectly suited for monitoring by NIRS, covered only by thin layers of skin, scalp, skull, and cerebrospinal fluid, and the word "promising" was used repeatedly in the literature that followed. A search on PubMed using the terms "NIRS or near-infrared" and "newborn or preterm" now yields 982 entries. While the hemoglobin oxygenation signal has achieved a high degree of construct validity, this is still being worked out for the cytochrome $\mathrm{aa}_{3}$ signal. $^{4}$

\subsection{Problem of Quantification}

The cerebral hemoglobin signals have been used to study a large variety of clinical questions. In this they have rarely produced surprises, i.e., cerebral oxygenation and blood volume have changed in the direction expected, but unfortunately, the absolute values have often been inconsistent. As an example, using the data from the two publications quoted above reporting a

*Address all correspondence to: Gorm Greisen, E-mail: greisen@ rh.dk relation between $\mathrm{PaCO}_{2}$ and cerebral blood volume, widely different values are obtained ( 0.5 to 1.9 versus $0.07 \mathrm{~mL}$ per $\mathrm{kPa}$ ).

Nearly 100 reviews have been written on NIRS and the preterm brain to date. The focus of the present review is the potential for clinical use to improve the outcome of preterm infants, and thus builds on and expands a previous article..$^{5}$

We here describe a research agenda with three components: randomized clinical trials to test the clinical value, pathophysiologic studies to quantify the effectiveness of clinical interventions, and improved instrumentation. We will argue that these research fields should be explored in parallel, rather than in the logical sequence: instrumentation, effectiveness, and clinical benefit. The reason is that there is a risk that cerebral oximetryas many other diagnostic methods-will enter clinical routine without good evidence of clinical benefit, i.e., there is a window of opportunity, which must be used.

\section{Clinical Problem}

Prematurity is now the leading cause of death of children under 5 years of age worldwide. ${ }^{6}$ In Europe alone, 25,000 infants are born every year with a gestational age of less than 28 weeks, of which 5000 die.

Preterm infants often require intensive care to support their immature respiratory and cardiovascular system. This may be during the first few days of life when the transition from fetal to neonatal life takes place or during complications later in the neonatal period such as episodes of sepsis or necrotizing enterocolitis. Infants needing intensive care have higher risk of neurodevelopmental impairment. This fact suggests that there is room for better tailoring of this intensive care to the needs of the individual infant, e.g., by improved monitoring.

\subsection{Need for Improved Clinical Monitoring}

A particular problem is to strike the balance between too little and too much oxygen. After five large randomized controlled 
trials comparing target ranges for pulse oximetry of $86 \%$ to $89 \%$ and $91 \%$ to $95 \%$, it is still uncertain which is better. ${ }^{7}$ The likely reason for this is that arterial oxygen saturation as monitored by pulse oximetry is only one of the factors of significance for tissue oxygen sufficiency and the risk of tissue hypoxia. The other major factors are blood hemoglobin concentration and tissue blood flow on the delivery side and tissue oxygen consumption on the uptake side. While hemoglobin concentration is easily available in clinical routine, blood flow and oxygen consumption are not. This is why tissue oximetry is relevant as a direct measure of oxygen sufficiency.

The tissue oxygenation $\left(\mathrm{StO}_{2}\right)$ is the oxygen-hemoglobin saturation in the blood inside the blood vessels in that tissue. In case of hemorrhage, extravascular blood is also included. It can be measured by NIRS using a range of different methods, the description of which is outside the scope of this review.

\subsection{Case for Cerebral Oximetry}

The brain of the preterm infant is particularly vulnerable. Although modern neonatology has significantly improved the chances of survival, the risk of neurodevelopmental impairment remains high. As an example, the risk of cerebral palsy is 1 of 1000 in term born infants, 1 of 100 at 34 weeks gestation, and 1 of 10 at 26 weeks gestation, and in Europe alone 5000 infants with gestational age below 28 weeks survive with neurodevelopmental deficit after brain injury each year.

The most common forms of brain injury in preterm infants are diffuse ${ }^{8}$ and hypoxia-ischemia, as well as peroxidation operates in concert with a range of other etiologic factors. Oxygenation of different regions of the brain does not appear to differ in preterm infants. ${ }^{9}$ This means that simple one-channel monitoring of the oxygenation of any region of the brain is likely to provide information that has the potential to benefit the infant by tailoring the intensive care, i.e., by modifying ventilatory or circulatory support (Fig. 1).

\subsection{Current Status}

The SafeBoosC-II trial showed that it is indeed possible to reduce the burden of cerebral hypoxia in extremely preterm infants during the first 3 days of life when adding cerebral

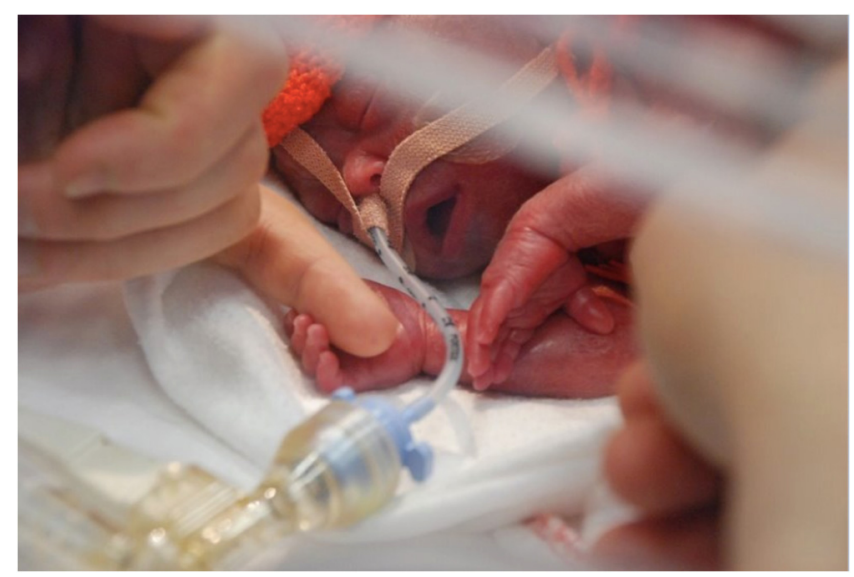

Fig. 1 The ultimate goal is to support preterm infants in an individualized manner during the physiological transition from fetal life. Due to their size and fragility all measures must be carefully adapted to their needs. Cerebral oximetry by NIRS has the potential to detect harmful cerebral hypoxia for timely intervention. oximetry to the already complex standard care. ${ }^{10}$ The burden was quantified as "the area under the curve" when the cerebral oxygenation was below a threshold of $55 \%$. The reduction was achieved by the use of continuous open-screen monitoring of cerebral oxygenation combined with a physiology-based treatment guideline suggesting how to intervene when the cerebral oximeter showed values outside the target range (Fig. 2). ${ }^{11}$ Perhaps surprisingly, many oximeter alarms were ignored, and most interventions only involved adjusting the fraction of inspired oxygen, i.e., using the cerebral oxygenation measure much like the reading from a pulse oximeter. But significant numbers of adjustments of the ventilatory and circulatory support were also recorded. ${ }^{12}$

The burden of cerebral hyperoxia was small and not influenced by interventions.

A similarly designed randomized clinical trial tested the value of monitoring of cerebral oxygenation in the delivery room during the first $15 \mathrm{~min}$ of life in preterm infants. Also in this trial the burden of cerebral hypoxia-as defined by a minute-to-minute reference curve-was reduced in the NIRSvisible group. ${ }^{13}$

\subsection{What Is to Be Done?}

The SafeBoosC trial discussed above involved 166 extremely preterm infants in eight university centers in Europe and was not powered to demonstrate an effect on clinically relevant outcomes such as brain injury, other complications, or survival, and did not do so. ${ }^{10,14,15}$ Similarly, the trial in the delivery room involved only 60 preterm infants and also did not demonstrate a clinical benefit. The problem is that while cerebral oximetry certainly has the potential for clinical benefit, it also has potential for harm. First, the sensors may injure the skin by heat and pressure. Second, adding another sensor and another cord to the already crowded and complex intensive care situation may confuse and take caregiver time from more important aspects of care, and use economic resources that could be spent better. Third, interventions that aim to improve cerebral oxygenation may have side effects.

A new, larger phase-III randomized trial is thus needed. A traditional RCT involving 1600 extremely preterm infants would have the power to detect a reduction in the risk of severe

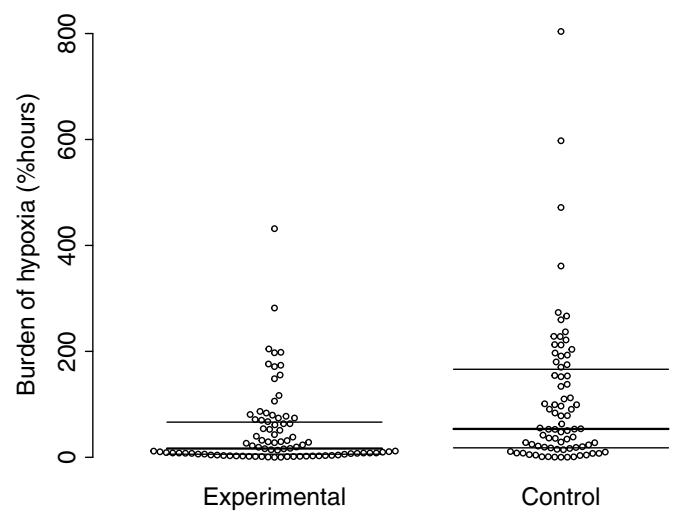

Fig. 2 The burden of cerebral hypoxia in 166 extremely preterm infants included in the SafeBoosC-II randomized clinical trial. The burden was reduced to less than half in the experimental group. The control group had blinded recording of cerebral oxygenation while the experimental group had the actual cerebral oxygen displayed and could act on cerebral hypoxia with the help of a clinical guideline. 
brain injury or death at 36 weeks of postmenstrual age from $34 \%$ to $27 \%$. Funding for such a trial was applied for by the SafeBoosC consortium in the EU Horizon2020 program, but unsuccessfully.

This leaves three likely scenarios. First, a large, pragmatic trial, perhaps cluster randomized, is realized to address the balance between benefit and harm in an effective way. Second, several smaller trials with similar interventions and outcomes are combined in a meta-analysis. Either way funding would partially have to be found locally. Third, as it is most common for new diagnostic and monitoring methods, cerebral oximetry will gradually enter clinical routine as driven by local professional ambition and marketing pressure without real evidence of benefit for patients and value for money for health providers. As examples, neither pulse oximetry nor transcutaneous monitoring of $\mathrm{pCO}_{2}$ has been evaluated in a stringent way in newborn infants. While pulse oximeters are in general use, this is not the case for transcutaneous monitoring of blood gases. More generally, new diagnostic methods may, apart from their own side effects and costs, potentially cause overdiagnosis and overtreatment. Currently, efforts are made to reduce what is perceived as unnecessary monitoring with pulse oximetry. ${ }^{16}$

It is a key problem that funding of formal testing of clinical benefit is not economically feasible for the industry and not required for approval of medical devices for clinical use.

\section{Clinical Research Problem}

Apart from the large-scale testing of the overall clinical benefit of monitoring of the cerebral oxygenation during intensive care, the value of individual interventions could be much better documented. While the ultimate goal is maximizing the chance of survival with a normal brain, improved knowledge of the pathophysiology that drives the poor outcomes is important for rational interventions.

\subsection{Better Definition of the Target Range for Cerebral Oxygenation}

For oxygenation really to enter everyday intensive care as a monitor, it has to come with a display of the current value, the trend, and a visible and audible alarm. But to set the alarm, it is necessary to know the safe range.

The first problem in setting a safe range is that cerebral oxygenation is a poorly defined physiological quantity. The best description is a venous-weighted tissue hemoglobin-oxygen saturation, indicating that the proportion of venous to arterial blood in the signal is uncertain and possibly varying. As the value appearing on the cerebral oximeter also represents a poorly defined lump of tissue, it is unreasonable to expect a close quantitative relation to other, better-defined physiological measures. Therefore, true validation is not possible, and any target range must be defined by construct validity.

\subsection{Population-Based Reference Ranges}

Recently, reference ranges for the first 3 days of life in preterm infants divided into gestational age bands were published. ${ }^{17}$ For gestational age 24 to 27 weeks, the 10th centile was around 55\% using the INVOS device with the adult sensor. These reference ranges were based on a very large number of infants. Differences were detected depending on the day after birth, gestational age by week, sex, and whether the infant was normally grown or small for gestational age. Although the differences were small to moderate of size, their statistical significance was great due to the large number of infants involved.

Unfortunately, reference ranges do not define optimality. The reference range for cerebral oxygenation is lower for infants with gestational age 24 to 25 weeks compared to infants with gestational age 30 to 31 weeks, but their risk of brain injury is also higher, and does the lower cerebral oxygenation play a role in this?

\subsection{Experimentally Based Hypoxic Thresholds}

In the newborn pig, experimental evidence shows that a threshold of 55\% includes a safety margin to allow almost all animals to maintain normal cerebral lactate and ATP, ${ }^{18}$ while it takes $30 \mathrm{~min}$ at a cerebral oxygenation of $35 \%$ to cause mitochondrial damage $^{19}$ and more than $2 \mathrm{~h}$ to cause neuronal death. ${ }^{20}$ As the pig brain is relatively mature at birth, this work should be repeated in animal models with brain maturation corresponding to extremely preterm infants and explore the effects of repetitive hypoxic insults, and the use of markers of milder damage and of damage to white matter.

\subsection{Safe Ranges Based on Association with Brain Injury}

Linking cerebral oxygenation to brain injury diagnosed in the neonatal period or neurodevelopmental impairment at followup has been attempted in several studies. "Fluctations" in cerebral oxygenation is associated with brain hemorrhage. ${ }^{21}$ Perhaps more significantly, lower levels of cerebral oxygenation have been associated with poor brain outcomes. ${ }^{22-26}$ Future work should use a predefined strategy for data analysis, e.g., drawing a receiver-operating curve for the prediction of severe brain injury for varying levels of cerebral hypoxic burden. This could help the construction of the rationale for one rather than another threshold for intervention. Also, future work should focus on timing. For cerebral hypoxia to cause brain injury it has to precede-not follow-the injury. It is a further complication that after a serious hypoxic-ischemic insult a phase of cerebral hyperperfusion and hyperoxygenation follows $^{27}$ and it is a challenge to sort this out in the statistical analysis.

\subsection{Improved Rationale for Clinical Responses to Cerebral Hypoxia}

When cerebral oximetry is used clinically, the effect of an intervention to bring back cerebral oxygenation into the target range can be observed directly, and hence provide better evidence of effectiveness in that particular situation in that particular patient than any piece of research on a group of similar patients. This is the power of a clinical monitoring tool, as it allows real individualization of care.

For the choice of intervention in the first place, however, good understanding of physiology and pathophysiology is useful. While reducing mechanical ventilation in an infant with a slightly low $\mathrm{pCO}_{2}$ and cerebral hypoxia is uncontroversial, the use of a vasopressor in an infant with a slightly low arterial blood pressure and cerebral hypoxia is not. Two current trials address this issue. ${ }^{28,29}$ Similarly, a large project addresses the usefulness of inotropic drugs to support the immature heart, ${ }^{30}$ also in terms of cerebral oxygenation. 


\subsection{Difficult Case of Erythrocyte Transfusion}

Most interestingly, several studies have demonstrated increased cerebral oxygenation after blood transfusion in preterm infants, most recently in infants during the first day of life and occurring only in infants with a low pretransfusion cerebral oxygenation. ${ }^{31}$

The effects of blood transfusion are complex, involving blood oxygen-carrying capacity and blood viscosity on one side and venous pressure and filling of the heart on the other. In situations with sufficient oxygen delivery to the brain, transfusion will simply lead to cerebral vasoconstriction through metabolic regulation. In one study, the effects of transfusion on cerebral oxygenation as well as on brain blood flow by Doppler were measured. ${ }^{32}$ By combining oxygenation and blood flow data, transfusion appears to reduce brain oxygen consumption - a highly unlikely event.

So it is not entirely clear if the increased cerebral oxygenation measured after transfusion really represent an increased delivery-to-consumption balance. Other possible explanations could be a shift in the oxygen-hemoglobin dissociation, or a shift in the venous to arterial blood volume ratio. Future studies should include measures of cerebral blood flow and cardiac output to disentangle the mechanisms to pave the way for an individualized use of blood transfusions.

\subsection{More Intelligent Use of Information on Cerebral Oxygenation}

The clinical trial of benefit and harm as described above will use cerebral oxygenation as a simple variable: an alarm will sound at a preset threshold and call for intervention. But even if this simple approach fails to deliver clinical benefit, it is possible that a more advanced approach could be useful, e.g., by combining absolute levels, trends, past reactions to intervention, and other clinical data.

\section{Technical Problem}

\subsection{Precision}

Lack of precision is the first problem. Precision is the ability to reach the same result repeatedly, and thus is the basis of "reproducibility." In clinical practice, single values inform treatment decisions, whereas statistically significant results in research come from averages, and where imprecision is just one of many sources of variance.

The first study focusing on the precision of cerebral oximetry reported a standard deviation of measurement of $5.2 \%$ after repeated replacement of the probe on the head of stable preterm infants. ${ }^{33}$ This level of (im)precision has been confirmed by others. The precision after replacement on a solid homogenous phantom is typically less than $1 \%$, and the typical spontaneous variability over time in stable preterm infants is typically less than $2 \%$.

As there is little evidence to support the idea of significant variability of oxygenation in different brain regions, ${ }^{9}$ the most likely explanation for this stubborn imprecision is the optic heterogeneity of the preterm head. To address this problem, a spatially resolved device was developed that combined a selfcalibrating algorithm and simultaneous measurement from two adjacent regions. Four light-emitting diodes and two photodiodes were put in a hexagonal arrangement. Unexpectedly, this device did not produce better precision, possibly due to light piping as a result of imperfect contact with the relatively hard newborn head. ${ }^{34}$

A precision standard deviation of $5 \%$ corresponds to a repeatability coefficient of $14 \%$. This means that the value from a probe that has been moved, or is sitting on another infant, has to differ by more than $14 \%$ in order to be taken to indicate that cerebral oxygenation really differs with a certainty of $95 \%$. Fourteen percent is a great deal considering that the typical value is around $70 \%$ and the threshold for intervention is around $55 \%$.

It has therefore been proposed to rely on trends only for clinical use, i.e., to disregard the absolute value, most radically by the use of a zero-setting procedure. This is a way of circumventing the lack of precision, the lack of accuracy, as well as the uncertainty of the hypoxic threshold. Whereas this may be meaningful in awake patients before anesthesia for heart surgery, it is not for preterm infants during the transition from intrauterine to extrauterine life since there is no initial normal state. Also, replacement of the probe or movement during prolonged monitoring may result in the same precision problem.

\subsection{What Is to Be Done?}

Technical development to improve the replacement precision is welcome. Perhaps other technologies than spatially resolved spectroscopy may prove better. A reasonable goal would be a precision in the same order of magnitude as the spontaneous variability over time in stable patients, i.e., $2 \%$ to $3 \%$.

\subsection{Agreement}

Cerebral oxygenation is an ill-defined physiologic quantity and therefore true validation is not possible. What has been done is calibration to oxygenation of blood drawn from an artery and a "cerebral" vein, usually the jugular vein in humans and sometimes the superior sagittal sinus in experimental animals. First, this depends on the assumption of a rather arbitrary arterial-tovenous ratio; second, the venous sampling sites are not ideal compared to the tissue actually measured. While it is obvious that cerebral oxygen saturation cannot exceed arterial saturation, it may actually be lower than venous saturation in situations with significant cerebral flow-metabolism heterogeneity. ${ }^{35}$

A more modest goal is agreement between different devices. The problem is that different devices that are approved for clinical use give different values.

Is has been consistently shown that the sensors for neonatal/ pediatric use give $\sim 10 \%$ points higher readings as compared to sensors for adult use with the same device. ${ }^{36-38}$ While it may be appropriate to calibrate a probe for a specific patient group or even a specific organ of interest, it is not entirely clear how this calibration was made or if the result is closer to the "true" value.

Second, in studies on stable preterm infants, some devices with sensors intended for neonatal use have given values in the range between $65 \%$ and $80 \%$ in reasonable agreement, while others have not. ${ }^{36,39,40}$

Third, even devices that agree within the normal range may deviate significantly in the hypoxic range as shown in infants born at term. ${ }^{41}$ This is most important, since it is in the hypoxic range that values may-or may not—lead to change in clinical care. 


\subsection{What ls to Be Done?}

Randomized clinical trials with brain injury, neurodevelopmental impairment, or mortality as the outcome will depend on a good guess on an appropriate hypoxic threshold. The threshold is defined by combining what is known about the pathophysiology with a calibration of the device(s) used in the trial in the hypoxic range.

In the SafeBoosC-II trial, ${ }^{10}$ the hypoxic threshold was set at $55 \%$ using two different devices that were found to give reasonably similar baseline values and hypoxic responses to arterial occlusion in the adult forearm-INVOS with the adult sensor and NIRO 200NX. ${ }^{42}$ Ideally, any cerebral oximeter that is used in randomized trials to test clinical benefit is compared to one of these devices and a hypoxic threshold equivalent to $55 \%$ is chosen.

For such comparison, a blood-lipid-yeast phantom may be used in which scatter, hemoglobin concentration, and oxygenation can be varied. Such a phantom is able to detect difference in calibration between devices with good precision ${ }^{43}$ and this data can be combined with data from infants. The goal is to reduce the uncertainty as regards the calibration of the hypoxic threshold to less than $5 \%$.

The ultimate goal is a trial with large-scale application of cerebral oximetry in the intensive care of preterm infants. The sensors therefore also need to be simple to apply, robust to the turmoil of intensive care, and affordable to hospitals around the world.

\section{Conclusion}

Routine clinical application of cerebral oximetry is in sight, but there are a number of issues to be solved. Large-scale clinical trials with patient relevant outcomes, better definition of hypoxic thresholds, and calibration of devices approved for clinical use. These issues can be addressed in parallel.

\section{Acknowledgments}

The Danish Strategic Research Council (Grant No. 060300482B) provided economical support for the SafeBoosC-II study. The photo in Fig. 1 was taken by the father and donated by the parents.

\section{References}

1. J. E. Brazy et al., "Noninvasive monitoring of cerebral oxygenation in preterm infants: preliminary observations," Pediatrics 75(2), 217-225 (1985).

2. J. S. Wyatt et al., "Quantification of cerebral oxygenation and haemodynamics in sick newborn infants by near infrared spectrophotometry," Lancet 328(8515), 1063-1066 (1986).

3. O. Pryds et al., "Carbon dioxide-related changes in cerebral blood volume and cerebral blood flow in mechanically ventilated preterm neonates: comparison of near infrared spectrophotometry and 133Xenon clearance," Pediatr. Res. 27(5), 445-449 (1990).

4. A. Bainbridge et al., "Brain mitochondrial oxidative metabolism during and after cerebral hypoxia-ischemia studied by simultaneous phosphorus magnetic-resonance and broadband near-infrared spectroscopy," Neuroimage 102 Pt 1, 173-183 (2014).

5. G. Greisen, T. Leung, and M. Wolf, "Has the time come to use near-infrared spectroscopy as a routine clinical tool in preterm infants undergoing intensive care?" Philos. Trans. A Math. Phys. Eng. Sci. 369(1955), 4440-4451 (2011).

6. L. Liu et al., "Child Health Epidemiology Reference Group of WHO and UNICEF. Global, regional, and national causes of child mortality: an updated systematic analysis for 2010 with time trends since 2000 ," Lancet 379(9832), 2151-2161 (2012).

7. V. Manja, S. Lakshminrusimha, and D. J. Cook, "Oxygen saturation target range for extremely preterm infants: a systematic review and meta-analysis," JAMA Pediatr. 169(4), 332-340 (2015).

8. J. J. Volpe, "Brain injury in premature infants: a complex amalgam of destructive and developmental disturbances," Lancet Neurol. 8, 110124 (2009).

9. R. G. Wijbenga, P. M. Lemmers, and F. van Bel, "Cerebral oxygenation during the first days of life in preterm and term neonates: differences between different brain regions," Pediatr. Res. 70(4), 389-394 (2011).

10. S. Hyttel-Sorensen et al., "Cerebral near infrared spectroscopy oximetry in extremely preterm infants: phase II randomised clinical trial," $B M J$ 350, g7635 (2015).

11. A. Pellicer et al., "The SafeBoosC phase II randomised clinical trial: a treatment guideline for targeted near-infrared-derived cerebral tissue oxygenation versus standard treatment in extremely preterm infants," Neonatology 104(3), 171-178 (2013).

12. J. Riera et al., "The SafeBoosC phase II clinical trial: an analysis of the interventions related with the oximeter readings," Arch. Dis. Child. Fetal. Neonatal. Ed. (2015).pii: fetalneonatal-2015-308829

13. G. Pichler et al., "Cerebral oxygen saturation to guide oxygen delivery in preterm neonates for the immediate transition after birth: a 2-center randomized controlled pilot feasibility trial," J. Pediatr. 170, 73-78.e4. (2016).

14. A. M. Plomgaard et al., "Brain injury in the international multicentre randomised SafeBoosC phase II feasibility trial: cranial ultrasound and magnetic resonance imaging assessments," Pediatr. Res. 79(3), 466472 (2016).

15. A. M. Plomgaard, "The SafeBoosC II randomised trial: treatment guided by near-infrared spectroscopy reduces cerebral hypoxia without changing early biomarkers of brain injury," Pediatr. Res. (2015) [Epub ahead of print].

16. A. C. Schondelmeyer et al., "Using quality improvement to reduce continuous pulse oximetry use in children with wheezing," Pediatrics 135(4), e1044-e1051 (2015).

17. T. Alderliesten et al., "Reference values of regional cerebral oxygen saturation during the first 3 days of life in preterm neonates," Pediatr. Res. 79(1-1), 55-64 (2016).

18. C. D. Kurth, W. J. Levy, and J. McCann, "Near-infrared spectroscopy cerebral oxygen saturation thresholds for hypoxia-ischemia in piglets," J. Cereb. Blood Flow Metab. 22(3), 335-341 (2002).

19. X. Hou et al., "Research on the relationship between brain anoxia at different regional oxygen saturations and brain damage using near-infrared spectroscopy," Physiol. Meas. 28, 1251-1265 (2007).

20. C. D. Kurth et al., "Cerebral oxygen saturation-time threshold for hypoxicischemic injury in piglets," Anesth. Analg. 108(4), 1268-1277 (2009).

21. C. M. Kissack et al., "Postnatal changes in cerebral oxygen extraction in the preterm infant are associated with intraventricular hemorrhage and hemorrhagic parenchymal infarction but not periventricular leukomalacia," Pediatr. Res. 56(1), 111-116 (2004).

22. L. C. Sorensen et al., "Neonatal cerebral oxygenation is not linked to foetal vasculitis and predicts intraventricular haemorrhage in preterm infants," Acta Paediatr. 97(11), 1529-1534 (2008).

23. E. A. Verhagen et al., "Cerebral oxygenation in preterm infants with germinal matrix-intraventricular hemorrhages," Stroke 41(12), 29012907 (2010).

24. K. K. Balegar et al., "Early cerebral oxygen extraction and the risk of death or sonographic brain injury in very preterm infants," J. Pediatr. 164(3), 475-480 (2014).

25. T. Alderliesten et al., "Hypotension in preterm neonates: low blood pressure alone does not affect neurodevelopmental outcome," J. Pediatr. 164(5), 986-991 (2014).

26. N. Baik et al., "Cerebral haemorrhage in preterm neonates: does cerebral regional oxygen saturation during the immediate transition matter?" Arch. Dis. Child. Fetal Neonatal Ed. 100(5), F422-F427 (2015).

27. G. Greisen, "Cerebral blood flow and oxygenation in infants after birth asphyxia," Early Hum. Dev. 90(10), 703-705 (2014).

28. E. M. Dempsey et al., "HIP Consortium. Management of hypotension in preterm infants (The HIP Trial): a randomised controlled trial of hypotension management in extremely low gestational age newborns," Neonatology 105(4), 275-281 (2014). 
29. P. Lemmers, "Clinical trials database," https://www.clinicaltrials.gov/ ct2/show/NCT01434251?term=tohop\&rank=1 (May 2015).

30. The NEO-CIRC project, http://neocirculation.eu (1 January 2016).

31. C. C. Andersen et al., "Early red cell transfusion favourably alters cerebral oxygen extraction in very preterm newborns," Arch. Dis. Child. Fetal Neonatal Ed. 100(5), F433-F435 (2015).

32. C. Dani et al., "Blood transfusions increase cerebral, splanchnic, and renal oxygenation in anemic preterm infants," Transfusion 50(6), 1220-1226 (2010).

33. L. C. Sorensen and G. Greisen, "Precision of measurement of cerebral tissue oxygenation index using near-infrared spectroscopy in preterm neonates," J. Biomed. Opt. 11(5), 054005 (2006).

34. S. Hyttel-Sorensen et al., "A comparison between two NIRS oximeters (INVOS, OxyPrem) using measurement on the arm of adults and head of infants after caesarean section," Biomed. Opt. Express 5(10), 3671-3683 (2014).

35. C. Metz et al., "Monitoring of cerebral oxygen metabolism in the jugular bulb: reliability of unilateral measurements in severe head injury," J. Cereb. Blood Flow Metab. 18(3), 332-343 (1998).

36. L. M. Dix et al., "Comparing near-infrared spectroscopy devices and their sensors for monitoring regional cerebral oxygen saturation in the neonate," Pediatr. Res. 74(5), 557-563 (2013).

37. A. Dullenkopf et al., "Measurement of cerebral oxygenation state in anaesthetized children using the INVOS 5100 cerebral oximeter," Pediatr. Anesth. 13(5), 384-391 (2003).

38. S. Hyttel-Sørensen et al., "Calibration of a prototype NIRS oximeter against two commercial devices on a blood-lipid phantom," Biomed. Opt. Express 4(9), 1662 (2013).
39. A. Schneider et al., "Comparison of four near-infrared spectroscopy devices shows that they are only suitable for monitoring cerebral oxygenation trends in preterm infants," Acta Paediatr. 103(9), 934-938 (2014).

40. T. Szczapa et al., "Comparison of cerebral tissue oxygenation values in full term and preterm newborns by the simultaneous use of two nearinfrared spectroscopy devices: an absolute and a relative trending oximeter," J. Biomed. Opt. 18(8), 87006 (2013).

41. T. W. Hessel, S. Hyttel-Sorensen, and G. Greisen, "Cerebral oxygenation after birth-a comparison of INVOS ${ }^{\circledR}$ and FORE-SIGHT ${ }^{\mathrm{TM}}$ near-infrared spectroscopy oximeters," Acta Paediatr. 103(5), 488-493 (2014).

42. S. Hyttel-Sorensen et al., "Tissue oximetry: a comparison of mean values of regional tissue saturation, reproducibility and dynamic range of four NIRS-instruments on the human forearm," Biomed. Opt. Express 2(11), 3047-3057 (2011).

43. S. Hyttel-Sorensen et al., "Calibration of a prototype NIRS oximeter against two commercial devices on a blood-lipid phantom," Biomed. Opt. Express 4(9), 1662-1672 (2013).

Gorm Greisen is a consultant neonatologist at Rigshospitalet and a professor of pediatrics at the University of Copenhagen. He defended his doctoral thesis 25 years ago on cerebral blood flow in mechanically ventilated infants and he has been active in this research field until now, culminating in the SafeBoosC-II trial with the ambition of taking a practical measure of oxygen sufficiency of the brain for use in the clinical care of preterm infants.

Biographies for the other authors are not available. 